\section{BRAZIULIAN JOURNAL}

OF MEDICAL AND BIOLOGICAL RESH ARCH

www.bjournal.com.br
ISSN 0100-879X

Volume 43 (01) 1-123 January 2010

BIOMEDICAL SCIENCES

AND

CLINICAL INVESTIGATION

Braz J Med Biol Res, J anuary 2010, Volume 43(1) 57-67

Immune cells and oxidative stress in the endotoxin tolerance mouse model

E.S. Melo, H.V. Barbeiro, S. Ariga, T. Goloubkova, R. Curi, I.T. Velasco, D. Vasconcelos and

F.G. Soriano

The Brazilian Journal of Medical and Biological Research is partially financed by
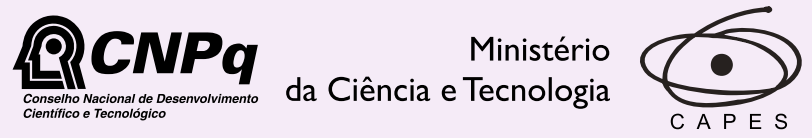

Ministério da Educação

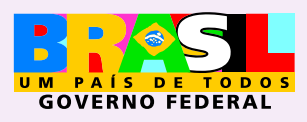

Institutional Sponsors 


\title{
Immune cells and oxidative stress in the endotoxin tolerance mouse model
}

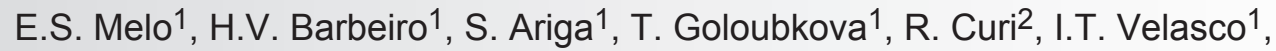 \\ D. Vasconcelos ${ }^{3}$ and F.G. Soriano ${ }^{1}$ \\ ${ }^{1}$ Disciplina de Emergências Clínicas, Faculdade de Medicina, \\ 2Departamento de Fisiologia e Biofísica, Instituto de Ciências Biomédicas, and \\ ${ }^{3}$ Laboratório de Investigação em Dermatologia e Imunodeficiência, \\ Departamento de Dermatologia, Instituto de Medicina Tropical, \\ Universidade de São Paulo, São Paulo, SP, Brasil
}

\begin{abstract}
Sepsis is a systemic inflammatory response that can lead to tissue damage and death. In order to increase our understanding of sepsis, experimental models are needed that produce relevant immune and inflammatory responses during a septic event. We describe a lipopolysaccharide tolerance mouse model to characterize the cellular and molecular alterations of immune cells during sepsis. The model presents a typical lipopolysaccharide tolerance pattern in which tolerance is related to decreased production and secretion of cytokines after a subsequent exposure to a lethal dose of lipopolysaccharide. The initial lipopolysaccharide exposure also altered the expression patterns of cytokines and was followed by an 8- and a 1.5-fold increase in the T helper 1 and 2 cell subpopulations. Behavioral data indicate a decrease in spontaneous activity and an increase in body temperature following exposure to lipopolysaccharide. In contrast, tolerant animals maintained production of reactive oxygen species and nitric oxide when terminally challenged by cecal ligation and puncture (CLP). Survival study after CLP showed protection in tolerant compared to naive animals. Spleen mass increased in tolerant animals followed by increases of B lymphocytes and subpopulation Th ${ }_{1}$ cells. An increase in the number of stem cells was found in spleen and bone marrow. We also showed that administration of spleen or bone marrow cells from tolerant to naive animals transfers the acquired resistance status. In conclusion, lipopolysaccharide tolerance is a natural reprogramming of the immune system that increases the number of immune cells, particularly T helper 1 cells, and does not reduce oxidative stress.
\end{abstract}

Key words: Sepsis; Cytokines; Immune tolerance; Lipopolysaccharide; Murine sepsis model; T helper cells

\section{Introduction}

Lipopolysaccharide (LPS) is one of the most potent known inducers of inflammation and exerts its effects via the Toll-like receptor (TLR) 4, which is essential for the recognition of distinct bacterial cell wall components (1-3). Inhibition of TLR signaling by inducible negative regulators, anti-inflammatory cytokines and alterations in the TLR signaling complex has been attributed to the phenomenon of "LPS tolerance" (1). Endotoxin tolerance has been defined as reduced responsiveness to a LPS challenge following a first encounter with endotoxin $(4,5)$. Tolerance to LPS involves the participation of macrophages and mediators such as glucocorticoids, prostaglandins, interleukin-10 (IL10), and transforming growth factor beta (4). However, the assumption that LPS tolerance is caused by a "generalized" down-regulation of cellular responses and mediator release is not accurate $(4,6)$. A similar loss of LPS reactivity has been repeatedly reported in circulating leukocytes of septic patients $(4,7)$. Studies of cellular signaling within leukocytes from septic patients have revealed numerous alterations similar to those observed in endotoxin-tolerant cells $(4,7)$. However, the altered responsiveness to LPS of leukocytes from septic patients is not synonymous with LPS tolerance. The term "cellular reprogramming" is helpful in describing the immune status of circulating leukocytes during the establishment of endotoxin tolerance $(4,8)$. Tolerance to LPS has been shown to protect against bacterial infections; it is a model of an adequate immune response in contrast to the deadly dysregulation of immune function in sepsis (4). In other studies, monophosphoryl lipid A has been used to produce tolerance and has been shown to protect animals

Correspondence: F.G. Soriano, Laboratório da Disciplina de Emergências Clínicas, FM, USP, Av. Dr. Arnaldo, 455, Sala 3187, 01246-913 São Paulo, SP, Brasil. Fax: +55-11-3061-7170. E-mail: gsoriano@usp.br

Received February 13, 2009. Accepted November 26, 2009. Available online December 18, 2009. Published January $11,2010$. 
from subsequent infections $(4,9)$.

Animal models represent a critical research bridge between experimental results from the laboratory and findings at the clinical level. Any disease, or intoxication, of humans that is established experimentally in animals should ideally mimic the natural sequelae seen in humans. The establishment of simple, economical, and robust animal models that can be used in different laboratories should facilitate the discovery of vaccines or therapies. One goal of the proposed murine model is to accelerate the development of vaccines or therapies that target a select family of bacterial protein toxins.

The aim of the present study was to evaluate a murine model of LPS tolerance. We characterized the complex cellular alterations associated with tolerance, including increased lymphocyte, monocyte and neutrophil proliferation.

\section{Material and Methods}

The present study was approved by the Research Ethics Committee of the University of São Paulo School of Medicine (protocol \#331/06). All animals were treated according to institutional rules for laboratory animal care.

Unless otherwise indicated, reagents were purchased from Sigma-Aldrich (USA).

\section{Induction of LPS tolerance}

Six-week-old male BALB/c mice weighing 20 to $25 \mathrm{~g}$ were randomized into two groups. The naive group ( $\mathrm{N}=$ $47)$ received daily subcutaneous injections of $1 \mathrm{~mL}$ saline, and the tolerant group ( $N=47)$ received daily subcutaneous injections of either 0.5 or $1 \mathrm{mg} / \mathrm{kg}$ LPS [Sigma-Aldrich (LPS Escherichia coli serotype 026:B6) catalogue number L3755] for 5 days and was allowed to rest for 2 days.

\section{Lethal LPS challenge}

Following induction of LPS tolerance and a 2-day rest, on day 8 some animals received either the lethal dose of LPS (20 mg/kg; $80 \%$ mortality in naive mice) or a supramaximal LPS dose (40 mg/kg; $100 \%$ mortality in naive mice) ip. Some animals from the tolerant and naive groups were sacrificed at 0 (zero), 2, 4, 6, and $8 \mathrm{~h}$ after the lethal challenges for biochemical measurements and cytokine analysis.

\section{Cecal ligation and puncture (CLP) model}

Mice were anesthetized with a mixture of ketamine (80 $\mathrm{mg} / \mathrm{kg}$ ) and xylazine $(10 \mathrm{mg} / \mathrm{kg}$ ) given intraperitoneally. Under aseptic conditions, a 2-cm midline laparotomy was performed to allow exposure of the cecum with adjoining intestine. The cecum was tightly ligated with a 3.0 silk suture (Ethicon; Johnson \& Johnson, USA) at its base, below the ileocecal valve, and was perforated twice (top and bottom) with an 18-gauge needle (Becton Dickinson, USA). The cecum was returned to the peritoneal cavity and the laparotomy was closed with 4.0 silk sutures. The animals were returned to their cages with free access to food and water.

\section{Study of animal survival}

Survival was assessed after CLP challenge or the administration of a lethal dose of LPS. Each group comprised 30 animals, and survival was assessed every $8 \mathrm{~h}$ up to $72 \mathrm{~h}$ after LPS or CLP. After $72 \mathrm{~h}$, there was no change in survival.

\section{Measurement of cytokines}

Blood was collected from 6 mice per group using tubes containing ethylenediamine tetraacetic acid $(1 \mathrm{mg} / \mathrm{mL})$. Blood was centrifuged, and the supernatant was harvested to identify secreted cytokines. Production of IL-6, IL-10, macrophage inflammatory protein 2 (MIP-2), tumor necrosis factor alpha (TNF- $\alpha$ ) and IL-1 $\beta$ was evaluated by enzymelinked immunosorbent assay (ELISA) using a DuoSet kit (R\&D Systems, USA). We performed all ELISA assays at the same time to avoid any kind of variation.

\section{Reverse transcriptase-polymerase chain reaction}

A reverse transcriptase-polymerase chain reaction (RT-PCR) assay was performed to measure the differential mRNA expression of TNF- $\alpha$, IL-10 and IL-1 $\beta$ in cells from naive and tolerant mice. Primer sequences were designed using information accessed from GenBank of the National Center for Biotechnology Information (NCBI, www.ncbi. nlm.nih.gov). For semi-quantitative PCR analysis, the housekeeping glyceraldehyde-3-phosphate dehydrogenase (GAPDH) gene sequence was used as a reference.

\section{PCR analysis}

The PCR products were analyzed on $1.5 \%$ agarose gels containing $0.5 \mu \mathrm{g} / \mathrm{mL}$ ethidium bromide and electrophoresed for $1 \mathrm{~h}$ at $100 \mathrm{~V}$. Gels were photographed using a Kodak DC120 Zoom Digital Camera System (Eastman Kodak, USA). Images were processed and analyzed with the Kodak Digital Science 1D Image Analysis software (Eastman Kodak). Band intensities were absorbance normalized with respect to GAPDH expression. Samples from naive mice $(\mathrm{N}=6)$ received an arbitrary value of 1 , and the values for samples from tolerant mice $(\mathrm{N}=6)$ were in relation to those for the respective naive animals.

\section{Thiobarbituric acid-reactive substances}

Formation of thiobarbituric acid-reactive substances (TBARS) was used to quantify the lipid peroxidation in tissues. Tissues were homogenized $(100 \mathrm{mg} / \mathrm{mL})$ in $1.15 \%$ $\mathrm{KCl}$. A total of $200 \mathrm{~mL}$ of the homogenates was then added to a reaction mixture consisting of $1.5 \mathrm{~mL} 0.8 \%$ thiobarbituric acid, $200 \mu \mathrm{L} 8.1 \%$ sodium dodecyl sulfate, $1.5 \mathrm{~mL} 20 \%$ acetic acid, $\mathrm{pH} 3.5$, and $600 \mu \mathrm{L}$ distilled water. The mixture was then heated to and maintained at $90^{\circ} \mathrm{C}$ for $45 \mathrm{~min}$. After 
cooling to room temperature, the samples were cleared by centrifugation at $10,000 \mathrm{~g}(10 \mathrm{~min})$ and their absorbance was measured at $532 \mathrm{~nm}$, using 1,1,3,3-tetramethoxypropane (Sigma-Aldrich) as an external standard. The level of lipid peroxides was reported as $\mu \mathrm{mol}$ TBARS/mg protein.

\section{Nitrite and nitrate}

Plasma nitrite and nitrate (NOx) levels were measured by the classic Griess method. After being passed through 50-kDa ultrafilters, $40 \mu \mathrm{L}$ plasma was diluted with $240 \mu \mathrm{L}$ assay buffer and mixed with $10 \mu \mathrm{L}$ cofactor and $10 \mu \mathrm{L}$ nitrate reductase (NOx colorimetric assay kit; Cayman Chemical, USA). After the plasma had been maintained at room temperature for $3 \mathrm{~h}$ to convert nitrate to nitrite, total nitrite was measured at $540 \mathrm{~nm}$ absorbance by reaction with Griess reagent (sulfanilamide and naphthalene-ethylene diamine dihydrochloride). Amounts of plasma nitrite were estimated by a standard curve obtained from enzymatic conversion of $\mathrm{NaNO}_{3}$ to nitrite.

\section{Spleen tissue preparation}

Spleen tissue $(50 \mathrm{mg}$ ) was pulverized in liquid nitrogen and homogenized in RIPA buffer $(10 \mathrm{mM}$ Tris- $\mathrm{HCl}$, $\mathrm{pH} 7.5), 1 \%$ tergitol, $0.1 \%$ sodium dodecyl sulfate, $1 \%$ Na-deoxycholate, $150 \mathrm{mM} \mathrm{NaCl}, 40 \mu \mathrm{g} / \mathrm{mL}$ phenylmethylsulfonyl fluoride, and $10 \mu \mathrm{g} / \mathrm{mL}$ pepstatin (Sigma, USA). Samples were centrifuged at $14,000 \mathrm{~g}$ for $45 \mathrm{~min}$ and protein concentrations from supernatants were determined by the Bradford method $(10,11)$.

\section{Quantification of leukocytes}

For flow cytometry analysis, spleen tissue was gently ground to dissociate cells. After hypotonic lysis of red blood cells, and following cell membrane permeabilization with saponin, leukocytes were incubated for $30 \mathrm{~min}$ at room temperature with fluorescein isothiocyanate-labeled antimouse monoclonal antibodies specific for murine CD3+ and CD4+ T-lymphocytes, and phycoerythrin-labeled antibodies to TNF- $\alpha$, interferon- $\mathrm{y}, \mathrm{IL}-10$, and IL-4. The analysis was performed using the Coulter EPICS XL-MCL flow cytometer (Beckman Coulter, UK) with gating on lymphocytes using characteristic forward and side scatter.

\section{Pluripotent cells}

The anti-apoptotic transcription factor Octamer-4 (Oct-4) is a member of the Pit-Oct-Unc family and a marker of pluripotent cells. The undifferentiated state of embryonic stem cells is characterized by a high level of Oct-4 expression. To determine levels of Oct-4, we used an embryonic stem cell recognition kit that includes antibodies to Oct-4 and SSEA1 (ES Cell Marker Sample Kit; Chemicon, USA). In brief, spleen cells were allowed to adhere to glass slides, after which they were washed twice with rinse buffer (TBST, 20 $\mathrm{mM}$ Tris- $\mathrm{HCl}, \mathrm{pH} 7.4,150 \mathrm{mM} \mathrm{NaCl}, 0.05 \%$ Tween 20). Cells were then fixed with paraformaldehyde and permeabilized with $0.1 \%$ Triton $\mathrm{X}-100$. Nonspecific markers were blocked with $5 \%$ bovine serum albumin, and the cells were incubated with primary antibody (anti-Oct-1 or anti-SSEA-1) at room temperature for $1 \mathrm{~h}$. After three washes in TBST, the cells were incubated with fluorescein isothiocyanate-conjugated secondary antibody for $30 \mathrm{~min}$. Cells were mounted on glass slides with antifade solution.

The slides were viewed under epifluorescence microscopy using a Zeiss Axioplan II microscope (Carl Zeiss, Germany) with a reticulated lens (100 grids of $1 \times 1 \mathrm{~mm}$ ). In each of 20 random fields, we counted the number of Oct-4- or SSEA-1-labeled cells among 200 nucleated cells, and the result is reported as the percent of the total. Three independent examiners performed the counts, and the final value represents the mean \pm SEM of those three measurements. The digital images were recorded using a cooled charge-coupled device camera (PCO Computer Optics, Germany), and analyzed using the ISIS program (MetaSystems, Germany). The final magnification was 100X.

\section{Transfer of tolerance}

Spleen tissue from tolerant mice was ground gently to dissociate cells. After hypotonic lysis of red blood cells and separation with Lymphoprep (Axis-Shield, Norway), leukocytes were resuspended in PBS buffer. Bone marrow cells were collected by flushing PBS into the marrow space. The collected mixture was resuspended in PBS for injection. During the first post-CLP hour, suspensions of cells from the spleen or bone marrow cells from tolerant or naive mice were injected intravenously into naive animals $(40,000$ cells/animal).

\section{Behavior and clinical parameters}

Mice were placed under anesthesia (3\% halothane for induction and $1.5 \%$ for maintenance in $30 \% \mathrm{O}_{2}$ and $70 \%$ $\mathrm{N}_{2} \mathrm{O}$ ). Using aseptic techniques, a temperature transmitter (E-mitter ${ }^{\circledR}$; Mini Mitter, USA) was placed in the peritoneal cavity via a midline incision. After surgery, mice were housed in individual cages, each with a running wheel and a telemetry system (Vitalview 4000 series; Mini Mitter). Temperature was monitored telemetrically. Signals were received by an antenna below the cage (ER-4000 Receiver; Mini Mitter) and relayed to a signal processor connected to a computer. Wheel revolutions were monitored magnetically and relayed to a signal processor connected to a computer. Measurements of mean temperature and accumulated wheel revolutions were collected every $1 \mathrm{~min}$.

\section{Statistical analysis}

Data were analyzed using the InStat software (GraphPad Software Inc., USA). Results are reported as means \pm SEM. Groups were compared by paired $t$-tests, as well as analysis of variance with the Tukey-Kramer post hoc test, when appropriate. For the study of survival, a log-rank test was used. Results were considered to be 
statistically significant at $P<0.05$.

\section{Results}

\section{Survival study}

The rate of survival following lethal LPS challenges was higher in animals that received prior treatment with LPS (tolerant) than in those not so treated (naive), as can be seen in Figure 1A,B. All animals received the lethal dose of LPS (either 20 or $40 \mathrm{mg} / \mathrm{kg}$ ). Overall survival for naive animals after $72 \mathrm{~h}$ was $10 \%$. Tolerant mice previously treated with $0.5 \mathrm{mg} / \mathrm{kg}$ LPS had a survival rate of $60 \%$, whereas animals that received treatment with $1 \mathrm{mg} / \mathrm{kg}$ LPS showed $100 \%$ survival compared to controls.

\section{Characterization of LPS tolerance}

In BALB/c mice, LPS induced tolerance in a dosedependent manner, as shown by the reduced mortality after a subsequent lethal dose of LPS (Figure 1A,B). In tolerant mice, production of proinflammatory cytokines (TNF- $\alpha$, IL-1 $\beta$ and IL-6; Figure 2A-C, respectively) and of the chemokine MIP-2 (Figure 2D) decreased in comparison to naive mice. In contrast, there was no difference in plasma levels of the anti-inflammatory cytokine IL-10 (Figure 2E). Levels of plasma $\mathrm{NO}$ and ROS (as measured via their NOx and TBARS products) remained unaffected in tolerant animals following LPS challenge (Figure 3A,B).

\section{Leukocyte analysis}

The spleen is the largest peripheral lymphatic organ and plays an important role in the inflammatory response. Following lethal LPS challenge $(20 \mathrm{mg} / \mathrm{kg})$, control $(\mathrm{N}=10)$ and tolerant $(\mathrm{N}=10)$ animals were submitted to splenectomy and the spleens were weighed. Tolerant animals presented higher spleen mass and a reduction in body weight relative to controls (Figure 4A,B).
Compared with those of controls, the spleens of tolerant animals contained larger numbers of $B$ cells and $T$ lymphocytes (CD4+ and CD8+), as shown in Figure 5A-F. The concentration of neutrophils in the spleen and blood was also higher in tolerant animals (data not shown). To determine whether a specific cell population was stimulated by LPS tolerance, T cells were analyzed by flow cytometry. Expression of TNF- $\alpha$, IFN- $\gamma$, IL-4, and IL-10 was measured from cytoplasm. An elevated number of $\mathrm{Th}_{1}$ (TNF- $\alpha$ and IFN-Y) lymphocytes $(P<0.05)$ was observed in tolerant compared to naive mice (Figure 5C-F). When animals were subjected to CLP, the spleens from the naive group exhibited a reduction in the number of cells containing TNF- $\alpha$ (Figure $5 \mathrm{C})$ compared with those from the LPS-tolerant group. In addition, tolerant animals presented higher numbers of CD4+ T cells expressing IFN-y (Figure 5E), IL-4 and IL-10 (Figure 5D,F) than did the controls. Compared with those of the controls, the spleens of tolerant animals also exhibited an increase in pluripotent cells that was maintained even after CLP challenge (Figure 6A). A significant reduction in mortality was observed when cells extracted from the spleens and bone marrow of tolerant mice were injected intravenously into naive animals previously subjected to CLP (Figure 6B). In contrast, the injection of spleen or bone marrow cells from naive animals did not confer any protection (Figure 6C).

\section{Clinical and behavioral change}

The body weight of the experimental animals was measured daily during induction of tolerance and after lethal LPS challenge. There was a continuous reduction in the weight of the animals after each LPS dose, as shown in Figure 4. Control animals showed no weight change during the first five days. After the lethal dose of LPS, both groups showed some reduction in body weight, which was greater in the control group. Temperature and spontaneous activity were
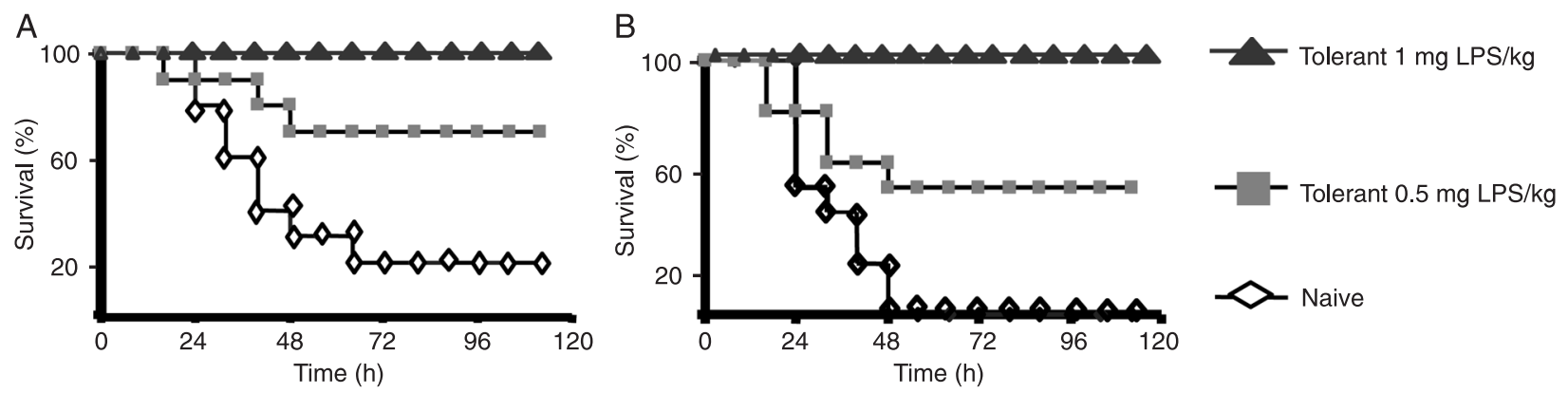

Figure 1. Lipopolysaccharide-induced tolerance in mice. BALB/c mice were treated daily with $0.5 \mathrm{or} 1 \mathrm{mg} / \mathrm{kg}$ lipopolysaccharide (LPS; tolerant group, $\mathrm{N}=30$ ) or saline placebo (naive group, $\mathrm{N}=30$ ) for 5 days. On day 8 , animals received either a lethal dose $(20 \mathrm{mg} / \mathrm{kg}$ ) or a supramaximal dose $(40 \mathrm{mg} / \mathrm{kg}$ ) of LPS. Animals tolerized by prior exposure to LPS had higher survival rates. A, Survival following a lethal dose of $20 \mathrm{mg} / \mathrm{kg}$ LPS. B, Survival following a supramaximal dose of $40 \mathrm{mg} / \mathrm{kg}$ LPS. Data are representative of 20 animals per group. 

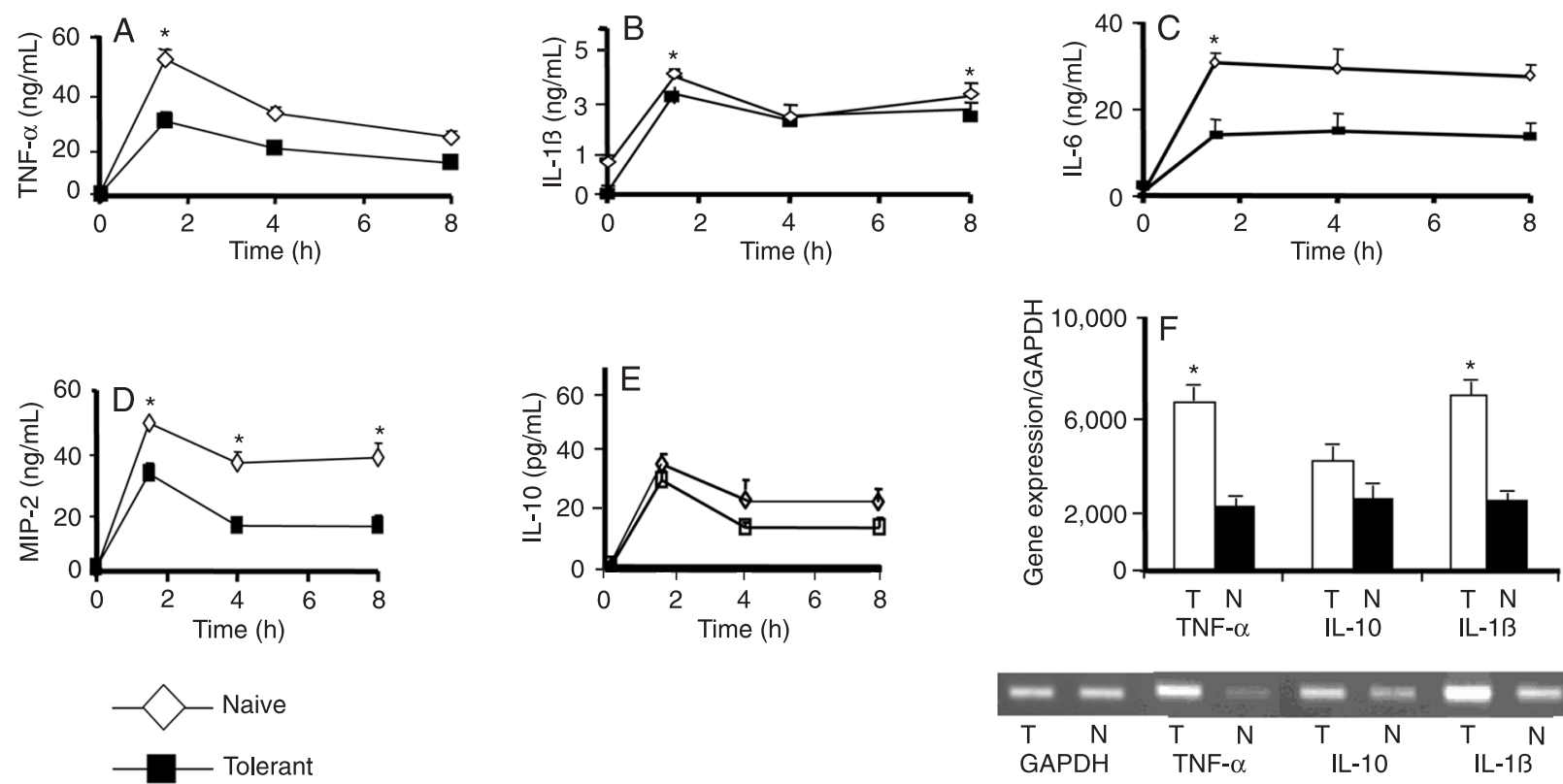

$\begin{array}{lllllll} & & & & & & \\ T & N & T & N & T & N & T\end{array}$

Figure 2. Cytokine production. BALB/c mice were treated with $1 \mathrm{mg} / \mathrm{kg}$ lipopolysaccharide (LPS; tolerant group, $\mathrm{N}=6$ ) or saline placebo (naive group, $\mathrm{N}=6$ ) daily for 5 days. Following a lethal dose of LPS $(20 \mathrm{mg} / \mathrm{kg})$ on day 8 , LPS-tolerant $(\mathrm{T})$ and naive $(\mathrm{N})$ animals were sacrificed at 0 (zero), 2, 4, 6, and $8 \mathrm{~h}$ to obtain cytokine data. Blood serum concentrations of cytokines: $A$, Tumor necrosis factor

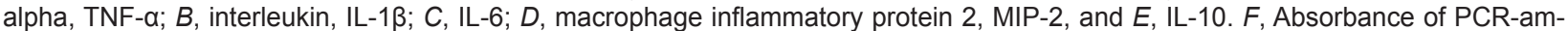
plified products (TNF- $\alpha, \mathrm{IL}-10$ and IL-1 $\beta$ ) are presented as the ratio cytokine/glyceraldehyde-3-phosphate dehydrogenase (GAPDH). Data are representative of 10 animals per group. ${ }^{*} \mathrm{P}<0.05$ compared to naive mice (analysis of variance with the Tukey-Kramer post hoc test).

A
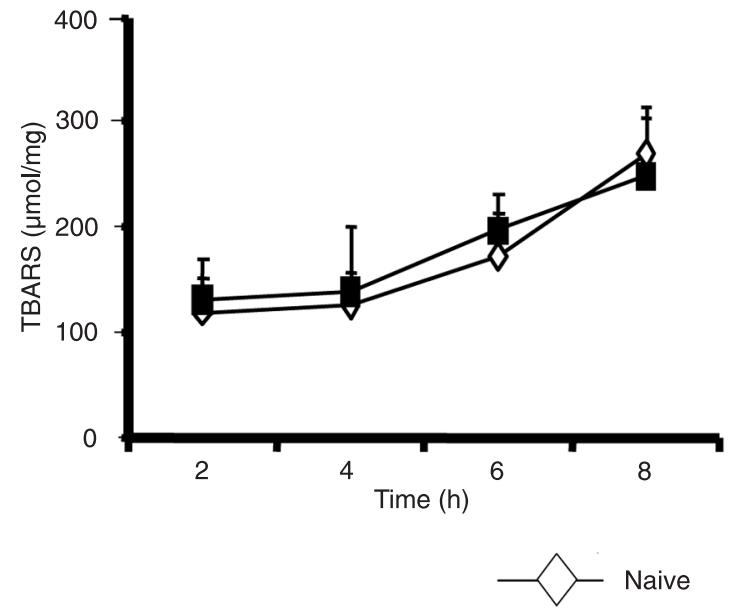

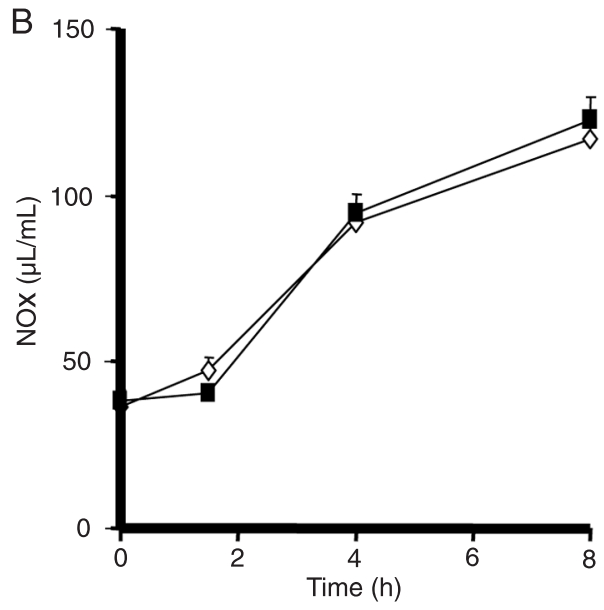

- Tolerant

Figure 3. Thiobarbituric acid-reactive substances (TBARS) and nitrite/nitrate production. Oxidative stress response and nitric oxide production in lipopolysaccharide-tolerant animals (tolerant group) and controls (naive group). $A$, TBARS per mg of protein was used as an index of oxidative stress. $B$, Blood plasma concentration of nitrite and nitrate (NOx). Data are representative of $\mathrm{N}=10$ animals per group. There were no significant statistical differences (analysis of variance with the Tukey-Kramer post hoc test). 

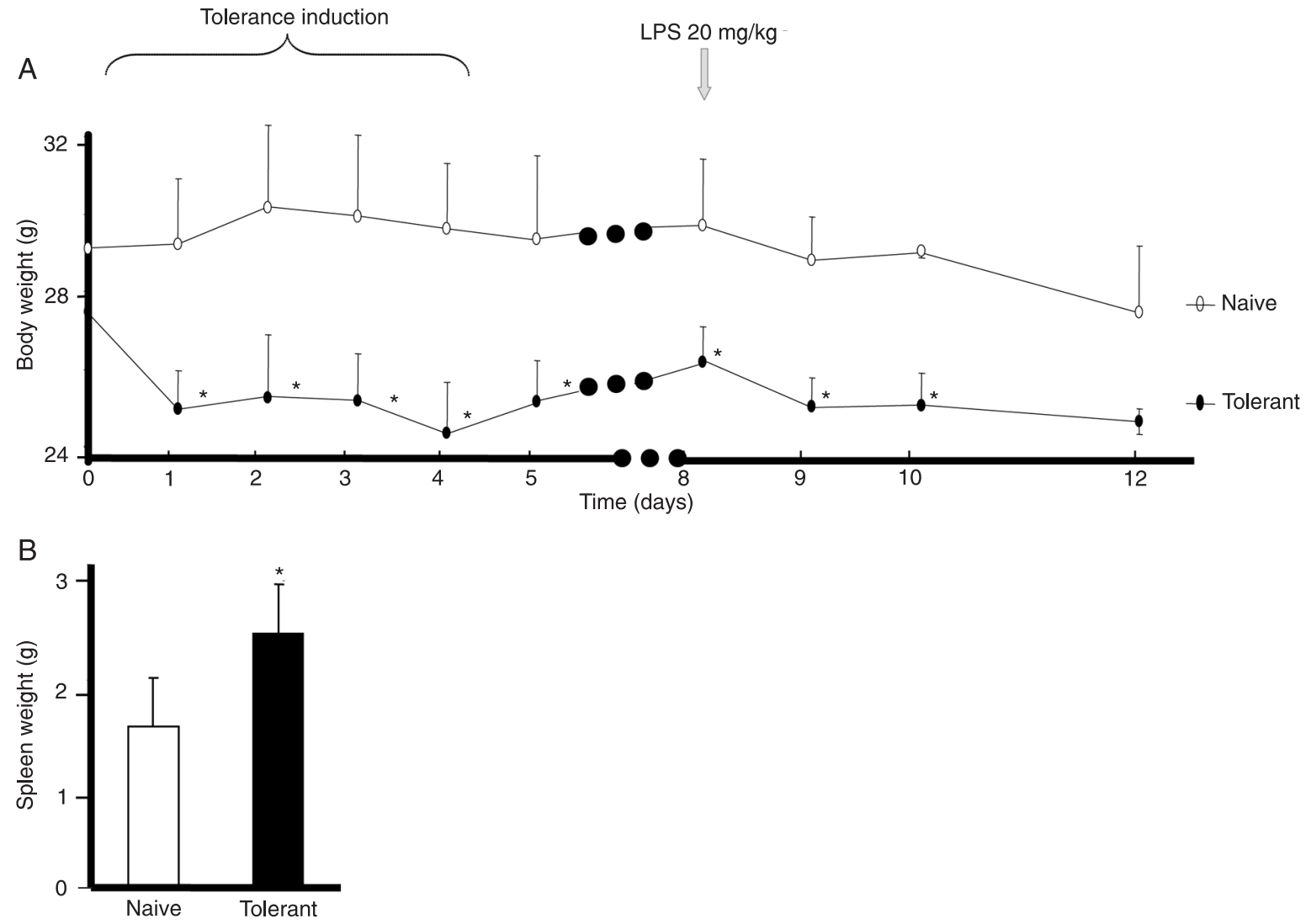

Figure 4. Body and spleen weight. Body and spleen weight of BALB/c mice treated with $1 \mathrm{mg} / \mathrm{kg}$ lipopolysaccharide (LPS; tolerant group) or saline placebo (naive group) daily for 5 days. Animals received a lethal dose of LPS (20 mg/kg) on day 8 , following a twoday rest. $A$, Change in daily body weight among tolerant and naive animals. Data are reported as means $\pm \mathrm{SEM}$. ${ }^{*} \mathrm{P}<0.05$ compared to naive mice (analysis of variance with the Tukey-Kramer post hoc test). $B$, Spleen weight of tolerant and naive animals. ${ }^{*} \mathrm{P}<0.05$ compared to naive mice (unpaired $t$-test).
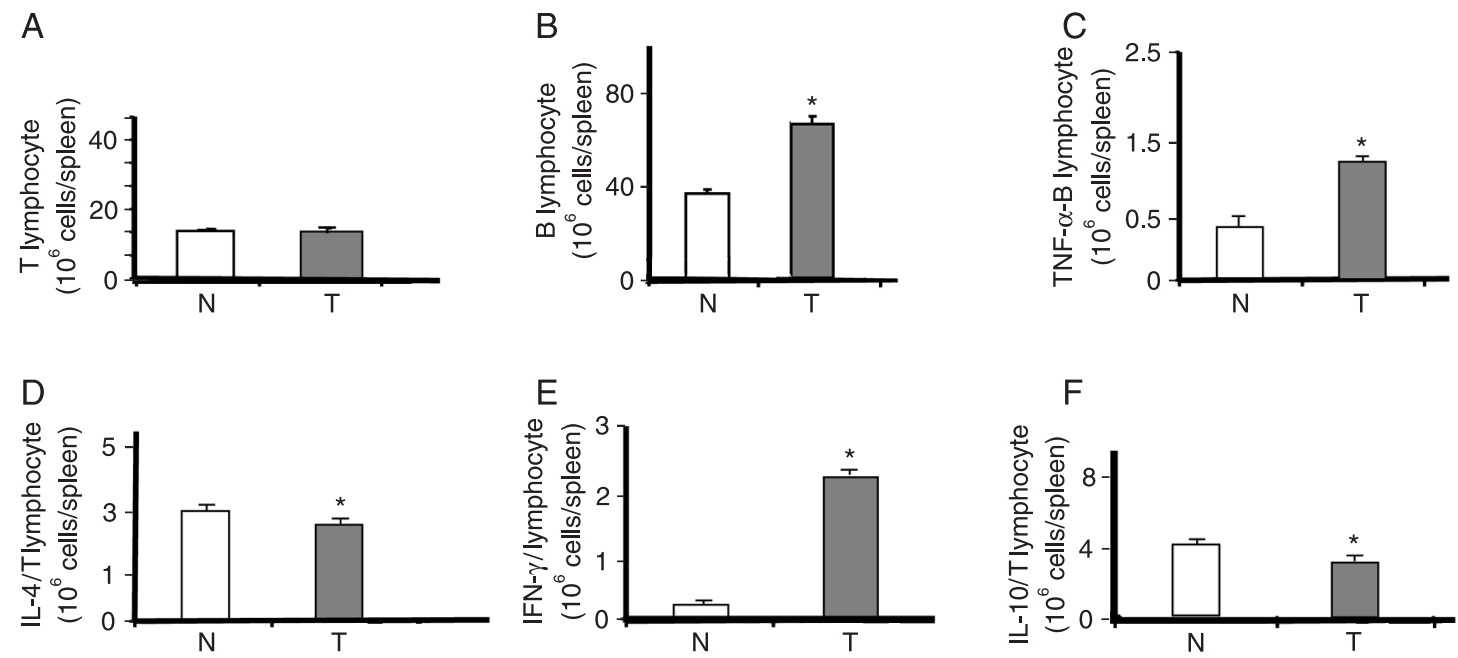

Figure 5. Quantitative analysis of spleen cells. Spleen lymphocyte counts in lipopolysaccharide-tolerant mice $(T)$ compared with naive mice (N) $6 \mathrm{~h}$ after cecal ligation and puncture for $A$, T lymphocytes; $B$, B lymphocytes; $C$, TNF-a-positive lymphocytes; $D$, IL-4-positive lymphocytes; $E$, interferon (IFN)-y-positive lymphocytes, and $F$, IL-10-positive lymphocytes. These data were obtained from a flow cytometry comparison of cells from tolerant and naive animals. ${ }^{*} \mathrm{P}<0.05$ compared to naive mice (unpaired $t$-test). 
A
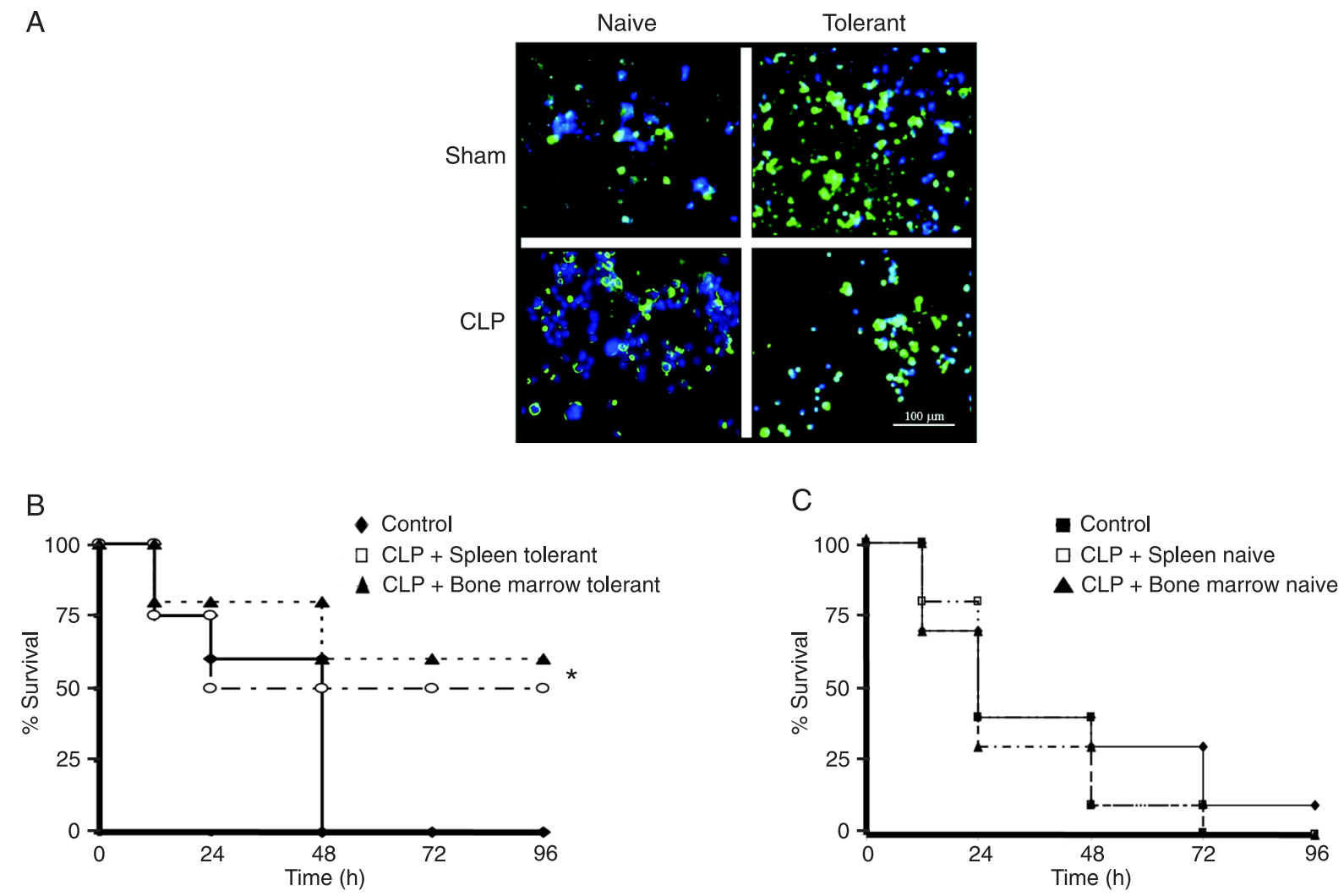

Figure 6. Pluripotent cells and transfer of tolerance. Suspensions of spleen or bone marrow cells from lipopolysaccharide-tolerant $\mathrm{BALB} / \mathrm{c}$ mice were injected intravenously in naive animals submitted to cecal ligation and puncture (CLP) or a sham procedure. $A$, Intensity of Oct-4 in a sample of pooled spleen and bone marrow cells from tolerant and naive mice under sham and CLP conditions (magnification, 100X). Mortality following CLP (B) was 100\% in naive mice after $48 \mathrm{~h}, 50 \%$ in naive mice injected with spleen cells after $72 \mathrm{~h}$, and $40 \%$ in naive mice injected with bone marrow cells after $72 \mathrm{~h}$. No protection against mortality following CLP was observed in a control group $(\mathrm{C})$ of naive mice that received cells suspended from the spleens or bone marrow of naive mice. Data are representative of 10 animals per group. ${ }^{*} \mathrm{P}<0.05$ for the difference between tolerant and naive groups (log-rank test).

measured throughout the period of LPS tolerance induction (Figure $7 \mathrm{~A}, \mathrm{~B}$ ). Temperature data showed a progressive increase in mean body temperature following each LPS dose. The temperature response when the lethal LPS dose (20 mg/kg) was applied was substantially higher than that observed for previous stimuli (Figure 7A). Spontaneous movement was measured by wheel activity. Animals showed a reduction in spontaneous activity during LPS tolerance induction and a large reduction after administration of the lethal dose of LPS (Figure 7B).

\section{Discussion}

A murine model of endotoxin tolerance offers an in vivo system for analyzing the regulatory feedback mechanisms involved in cytokine synthesis (5). Endotoxin tolerance has been divided into an early, non-immunological phase and a later immunological phase in which circulating antibodies might play a significant role in endotoxin detoxification
$(12,13)$. Based on literature data, we hypothesized that LPS tolerance produces an "adaptation" of the innate immune response (1). Previous studies have presented conflicting findings regarding cytokine production during lethal LPS challenges, as well as regarding the extent to which tolerance provides resistance to infection (4). Most of these differences are due to differences in the model used, doses used to establish tolerance, injection times, administration routes, and the time of delay before restimulation with LPS or infection $(4-6,14,15)$. In the present study, we established and characterized a reliable murine model of endotoxin tolerance in which we applied subcutaneous injection of LPS for inducing tolerance. While intraperitoneal injections are commonly reported in the literature, they cannot exclude the chance that protection against CLP is a local effect, perhaps activating local macrophages and the large number of neutrophils located in the peritoneal cavity $(4-6,14,15)$. Because stress produces glucocorticoids that down-regulate proinflammatory cytokines, we waited for 


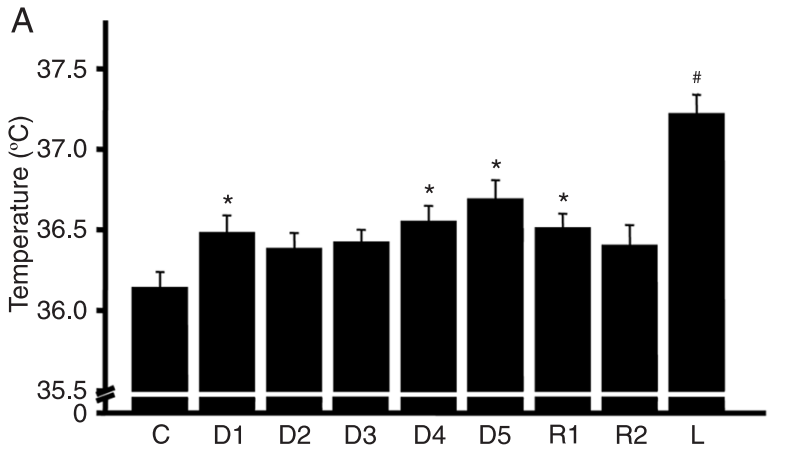

B

Figure 7. Effect of lipopolysaccharide (LPS) tolerance on body temperature and exercise. BALB/c mice $(\mathrm{N}=8)$ were injected daily (D1, D2, D3, D4, and D5) with $1 \mathrm{mg} / \mathrm{kg}$ LPS, followed by 2 days of rest (R1 and R2) and lethal (L) injection of LPS (20 mg/kg). Effect of treatment on (A) mean $24 \mathrm{~h}$ body temperature and (B) mean $24 \mathrm{~h}$ exercise wheel activity. Data are representative of 8 animals. ${ }^{*} \mathrm{P}$ $<0.05$ vs control group (C); ${ }^{P}<0.05$ vs all other groups; $\$ \mathrm{P}<0.05$ vs D1 and D4; ${ }^{+} \mathrm{P}<0.05$ vs D1-D5 (analysis of variance with the Tukey-Kramer post hoc test).

two days after establishing tolerance before challenging animals with LPS or CLP $(4,16)$. Our aim was to identify changes in cell populations and gene expression produced by tolerance and to discover novel protection mechanisms in addition to the known effects of glucocorticoids. Models that do not allow animals to rest prior to a re-exposure to LPS, and others in which CLP is performed during the cytokine storm and inflammatory response, can result in investigators analyzing immunosuppression or immunoparalysis rather than a true tolerance phenomenon $(4,5,10,11,15)$. In addition, we established tolerance using two doses of LPS and found that protection is dose-dependent.

\section{LPS tolerance model}

We report that LPS tolerance is associated with substantial and selective alterations in animal behavior and immune cells. In animals, LPS tolerance is a highly effective mechanism for protection against the lethal syndrome of severe sepsis and other diseases $(4,5)$. This phenomenon, which seemingly prepares the organism for a subsequent infection, was originally described as a form of "blunted endotoxin-associated mortality" not necessarily related to cytokine production (5). The first aim of our study was to confirm that our procedure produced a reliable model of LPS tolerance. This was a critical step, due to the variety of tolerance models in the literature using different LPS doses, frequencies and periods $(4,5)$. We established tolerance using two different doses of LPS. The mortality data demonstrate that the protective effect of LPS-induced tolerance is dose-dependent. A dose of $1 \mathrm{mg} / \mathrm{kg}$ LPS per day over 5 days provided complete protection in experimental animals, whereas a dose of $0.5 \mathrm{mg} / \mathrm{kg}$ per day provided only partial protection. We then measured the production of selected cytokines after animals had been challenged with a lethal dose of LPS. Because of economic and ethical constraints, cytokine analysis was performed using only the animals that achieved complete protection (i.e., the $1 \mathrm{mg} /$ $\mathrm{kg}$ group). We found that injection of lethal doses of LPS into mice results in transient increases in serum levels of TNF- $\alpha, \mathrm{IL}-1 \beta, \mathrm{IL}-10, \mathrm{IL}-6$, and MIP-2. The data show that induction of tolerance produced a significant reduction in the plasma levels of TNF- $\alpha$, IL-1 $1 \beta$, IL- 6 , and MIP-2, but not of IL-10. There are conflicting data in the literature regarding $\mathrm{IL}-1 \beta$; some investigators have reported that production of IL-1 $\beta$ decreases in tolerant animals, whereas others found no difference $(4,5)$. Our experiments showed that, in animals tolerized with a high LPS induction dose, IL-1 $\beta$ production decreased following administration of the lethal LPS dose. In addition, using our model, we found that tolerance induces a clear anti-inflammatory response, since IL-10 production was unchanged in tolerant animals and pro-inflammatory cytokines were reduced. Although a reduction in IL-10 production in tolerant animals during terminal LPS challenge has been described previously, some investigators have found either increases or no change $(4,5)$. These discrepancies require us to carefully characterize our LPS tolerance model. The anti-inflammatory cytokine IL-10 not only deactivates macrophages and neutrophils but also has the ability to alter the type and phase of the immune response (17). The diversity of biologic responses mediated by cytokines requires a fuller understanding of their regulation during homeostasis and in various pathological conditions.

We can avoid an intense inflammatory response using tools to induce a pre-existing state of tolerance. The inflammatory response evoked in order to eliminate bacteria is mediated by cytokines that govern the release of enzymes (e.g., metalloproteinases), as well as by those that regulate phagocytosis, oxidative stress, and nitrosative stress $(18,19)$. Proper immune function relies on anti-inflammatory cytokines to appropriately target physiological responses to bacteria. For instance, IL- 6 and IL-10 act on B cell differentiation and increase antibody production $(17,20,21)$, although appropriate amounts of anti- and pro-inflammatory 
cytokines are necessary (11). Sepsis progresses via an exacerbated inflammatory response, including tissue necrosis and leukocyte apoptosis $(11,22)$. Therefore, sepsis is an inappropriate strategy to eliminate invading microorganisms $(11,22)$. In our study, spleen volume increased in tolerant animals, which, as we showed using cell counts and cytometry, reflects an increased number of immune cells. Patients and animals deficient in specific immune cells are more susceptible to infection and death $(23,24)$. In addition, Hotchkiss and Karl (22) showed that apoptosis of lymphocytes and dendritic cells are associated with death from sepsis in human patients. This observation was confirmed in experimental studies in which apoptosis inhibition protected against polymicrobial infection (22). The increased number of lymphocytes and neutrophils in the spleens of tolerant animals in our study might signal a greater capacity on the part of the immune system to fight bacteria. In our LPS tolerance model, NOx and TBARS levels were not reduced in response to a lethal LPS challenge. Although production of NO in LPS tolerance has been controversial, Hirohashi and Morrison (25) showed that the release of NO depends on the tolerogenic LPS dose. We highlight here that protection against lethal LPS and CLP challenges found in LPS-tolerant animals is not caused by a reduction in NO. Despite some beneficial effects of NO inhibition, NO is a superoxide that the body uses to kill bacteria $(11,26)$. These data raise the question of whether treating septic patients with antioxidant therapy is appropriate, or whether it is better to block the terminal effects of sepsis, i.e., reducing apoptosis and necrosis $(11,22)$.

In order to demonstrate the clinical relevance of LPS tolerance, we performed mortality studies using CLP, an accepted polymicrobial model of sepsis. Eichacker et al. (27) showed that anti-inflammatory interventions in sepsis were successful when the control group presented mortality rates greater than $60 \%$. Lower mortality rates $(40 \%)$ in controls were associated with no detectable protection by the various anti-inflammatory strategies proposed as experimental therapeutic approaches for the treatment of sepsis (27). In clinical trials, mortality from sepsis is generally $30-40 \%$ (27). This might explain why approaches that produce positive results in experimental studies routinely fail in clinical trials (27). The protection against death conferred by LPS tolerance in our study was apparent not only in severe sepsis but also when mortality rates were lower in the control group. This suggests that LPS tolerance is effective in preventing death from sepsis in a wide range of disease severity. Some studies in the literature report that LPS tolerance provides protection against infections $(4,14,15)$. However, that finding has not been confirmed, and some investigators have detected an increase in mortality $(4,5)$. Comparing these studies is difficult because of the different models of tolerance, bacterial infection and fungal infection involved $(4,5,14,15)$. However, as in our study, a preponderance of more recent reports have shown that LPS tolerance offers a protective effect in sepsis $(4,5,14,15)$. We believe that the LPS tolerance model is characterized by changes in the immune system, and that these changes help it remain correctly regulated during sepsis. As a result, such models are not only useful for studying sepsis, but can point the way to new strategies for protecting patients. Although most studies in the literature have focused on ways to protect animals and patients by halting the clinical progression of sepsis, these strategies have failed to lower the mortality of sepsis (22).

\section{Spleen cells in LPS tolerance}

Differences in the qualitative and quantitative immune response to microbial stimulation might be critical for the understanding of the pathophysiology of sepsis $(5,18)$. Although T-cell dysfunction is known to occur in sepsis, the increased release of cytokines has received far more attention than have changes in T-cell populations (28). Here, we observed that the spleens of tolerant mice contained more lymphocytes, monocytes, neutrophils, and pluripotent cells than did those of controls. It is known that $\mathrm{T}$ cells express Toll-like receptors, and that CD4+ T cells produce key cytokines and chemokines that can activate and attract macrophages and neutrophils to infection sites (28). Defective T-cell proliferation and cytokine secretion correlates with increased mortality in sepsis (22). In our study, LPS tolerance was not accompanied by similar defects in T-cell activity. Our results show that tolerance induction leads to changes in macrophages and lymphocytes, particularly $\mathrm{T}$ cells. In tolerant animals, the $\mathrm{Th}_{1}$ population of $\mathrm{T}$ lymphocytes was elevated, as evidenced by the cells containing the markers TNF- $\alpha$ and IFN- $\gamma$, indicating that the lymphocytes were activated. These results might seem to contrast with our findings vis à vis cytokine levels in blood plasma. However, it is important to note that baseline cytokine levels were higher in tolerant animals than in controls. However, when the animals were submitted to CLP, the tolerant mice produced lower amounts of cytokines. These results suggest that the pre-activated lymphocytes were tempering the inflammatory response, as has been reported in the literature (29).

To determine whether immune cells previously exposed to LPS maintain the properties of tolerance in a new host, cells were removed from the spleens and bone marrow of tolerant mice and injected into naive animals after CLP challenge. Significantly lower mortality rates were observed among animals that received transfers of either cell type. This effect was not observed in control experiments in which bone marrow or spleen cells from naive animals was injected instead. The control experiments helped exclude possible nonspecific effects of cellular transfer. The pool of cells obtained from the spleens and bone marrow of tolerant mice contained elevated levels of Oct-4, which participates in the epigenetic reprogramming of somatic cells into embryonic stem cells and is also a marker for pluripotent cells 
$(30,31)$. Our findings show that LPS tolerance induced an increase in the number of pluripotent cells in the spleen. Other investigators recently reported that administering bone marrow stem cells to mice reduced mortality in a CLP model of sepsis (32). Stem cells are known to modulate the immune response and to decrease apoptotic events in neighboring cells, a critical and dangerous phenomenon seen in septic patients (32). Our data are insufficient to say whether the protective effect observed was related to the transfer of lymphocytes, monocytes or stem cells. Previously, Freudenberg and Galanos (33) and others $(2,4)$ showed that macrophage transfer protected animals from a lethal LPS dose. It is our view that no single cell type is able to transfer acquired protection to sepsis. In our study, cells were tolerized by exposure to LPS, and the physiology of naive animals was altered to protect them from CLP. These data show that tolerance alters the innate immune response of cells and that protection does not require the production of antibodies. The cells maintain a memory of exposure to LPS and when they are transferred to a naive animal the protective effect is maintained.

\section{References}

1. Foster SL, Hargreaves DC, Medzhitov R. Gene-specific control of inflammation by TLR-induced chromatin modifications. Nature 2007; 447: 972-978.

2. Poltorak A, He X, Smirnova I, Liu MY, Van HC, Du X, et al. Defective LPS signaling in $\mathrm{C} 3 \mathrm{H} / \mathrm{HeJ}$ and $\mathrm{C} 57 \mathrm{BL} / 10 \mathrm{ScCr}$ mice: mutations in Tlr4 gene. Science 1998; 282: 20852088.

3. Takeuchi O, Akira S. Toll-like receptors; their physiological role and signal transduction system. Int Immunopharmacol 2001; 1: 625-635.

4. Cavaillon JM, Adib-Conquy M. Bench-to-bedside review: endotoxin tolerance as a model of leukocyte reprogramming in sepsis. Crit Care 2006; 10: 233.

5. West MA, Heagy W. Endotoxin tolerance: A review. Crit Care Med 2002; 30: S64-S73.

6. Fan $\mathrm{H}$, Cook JA. Molecular mechanisms of endotoxin tolerance. J Endotoxin Res 2004; 10: 71-84.

7. Rigato O, Salomao R. Impaired production of interferongamma and tumor necrosis factor-alpha but not of interleukin 10 in whole blood of patients with sepsis. Shock 2003; 19: 113-116.

8. Cavaillon JM, Adrie C, Fitting C, Adib-Conquy M. Reprogramming of circulatory cells in sepsis and SIRS. J Endotoxin Res 2005; 11: 311-320.

9. Mata-Haro V, Cekic C, Martin M, Chilton PM, Casella CR, Mitchell TC. The vaccine adjuvant monophosphoryl lipid A as a TRIF-biased agonist of TLR4. Science 2007; 316: 16281632.

10. Liaudet L, Murthy KG, Mabley JG, Pacher P, Soriano FG, Salzman AL, et al. Comparison of inflammation, organ damage, and oxidant stress induced by Salmonella enterica serovar Muenchen flagellin and serovar Enteritidis lipopolysaccharide. Infect Immun 2002; 70: 192-198.

\section{Chronic infection model}

Chronic infections frequently cause reduction in body weight and an increase in spleen size, as well as slight increases in body temperature and reduced spontaneous physical activity. Most of these signs have been linked to persistently elevated levels of cytokine release (34). A similar pattern was observed in animals in our study during the period of tolerance induction. Given these results, we hypothesize that the period during which LPS tolerance is induced can be used as a model to study chronic infection and related metabolic and immunological alterations.

The present study sheds new light on the model of LPS tolerance and the immunological alterations that accompany tolerance, as well as showing that LPS tolerance can be used in the study of chronic infections.

\section{Acknowledgments}

This study was supported by FAPESP (\#02/07430-6) and CNPq (\#470744/2004-9).
11. Soriano FG, Liaudet L, Szabo E, Virag L, Mabley JG, Pacher $P$, et al. Resistance to acute septic peritonitis in poly(ADPribose) polymerase-1-deficient mice. Shock 2002; 17: 286292.

12. Greisman SE, Hornick RB. The nature of endotoxin tolerance. Trans Am Clin Climatol Assoc 1975; 86: 43-50.

13. Greisman SE, Young EJ, Workman JB, Ollodart RM, Hornick RB. Mechanisms of endotoxin tolerance. The role of the spleen. J Clin Invest 1975; 56: 1597-1607.

14. Rayhane N, Fitting C, Lortholary O, Dromer F, Cavaillon JM. Administration of endotoxin associated with lipopolysaccharide tolerance protects mice against fungal infection. Infect Immun 2000; 68: 3748-3753.

15. Wheeler DS, Lahni PM, Denenberg AG, Poynter SE, Wong $\mathrm{HR}$, Cook JA, et al. Induction of endotoxin tolerance enhances bacterial clearance and survival in murine polymicrobial sepsis. Shock 2008; 30: 267-273.

16. Flohe S, Dominguez FE, Ackermann M, Hirsch T, Borgermann J, Schade FU. Endotoxin tolerance in rats: expression of TNF-alpha, IL-6, IL-10, VCAM-1 and HSP 70 in lung and liver during endotoxin shock. Cytokine 1999; 11: 796-804.

17. Bonig $\mathrm{H}$, Packeisen J, Rohne B, Hempel L, Hannen M, Klein-Vehne A, et al. Interaction between interleukin 10 and interleukin 6 in human B-cell differentiation. Immunol Invest 1998; 27: 267-280.

18. Deng JC, Cheng G, Newstead MW, Zeng X, Kobayashi K, Flavell RA, et al. Sepsis-induced suppression of lung innate immunity is mediated by IRAK-M. J Clin Invest 2006; 116: $2532-2542$.

19. Kobayashi K, Hernandez LD, Galan JE, Janeway CA Jr, Medzhitov R, Flavell RA. IRAK-M is a negative regulator of Toll-like receptor signaling. Cell 2002; 110: 191-202.

20. Cerutti A, Zan H, Schaffer A, Bergsagel L, Harindranath N, 
Max EE, et al. CD40 ligand and appropriate cytokines induce switching to $\lg G$, $\lg A$, and $\lg E$ and coordinated germinal center and plasmacytoid phenotypic differentiation in a human monoclonal IgM+lgD+ B cell line. J Immunol 1998; 160: 2145-2157.

21. Duddy ME, Alter A, Bar-Or A. Distinct profiles of human B cell effector cytokines: a role in immune regulation? J Immunol 2004; 172: 3422-3427.

22. Hotchkiss RS, Karl IE. The pathophysiology and treatment of sepsis. N Engl J Med 2003; 348: 138-150.

23. Hayakawa K, Hardy RR, Herzenberg LA. Peritoneal Ly-1 B cells: genetic control, autoantibody production, increased lambda light chain expression. Eur J Immunol 1986; 16: 450-456.

24. Popi AF, Lopes JD, Mariano M. Interleukin-10 secreted by $\mathrm{B}-1$ cells modulates the phagocytic activity of murine macrophages in vitro. Immunology 2004; 113: 348-354.

25. Hirohashi N, Morrison DC. Low-dose lipopolysaccharide (LPS) pretreatment of mouse macrophages modulates LPSdependent interleukin-6 production in vitro. Infect Immun 1996; 64: 1011-1015.

26. Alves-Filho JC, de Freitas A, Spiller F, Souto FO, Cunha FQ. The role of neutrophils in severe sepsis. Shock 2008; 30 (Suppl 1): 3-9.

27. Eichacker $P Q$, Parent C, Kalil A, Esposito C, Cui X, Banks $\mathrm{SM}$, et al. Risk and the efficacy of antiinflammatory agents: retrospective and confirmatory studies of sepsis. Am J Respir Crit Care Med 2002; 166: 1197-1205.

28. Scumpia PO, Delano MJ, Kelly-Scumpia KM, Weinstein JS,
Wynn JL, Winfield RD, et al. Treatment with GITR agonistic antibody corrects adaptive immune dysfunction in sepsis. Blood 2007; 110: 3673-3681.

29. Kim KD, Zhao J, Auh S, Yang X, Du P, Tang H, et al. Adaptive immune cells temper initial innate responses. Nat Med 2007; 13: $1248-1252$.

30. Takahashi K, Tanabe K, Ohnuki M, Narita M, Ichisaka T, Tomoda $\mathrm{K}$, et al. Induction of pluripotent stem cells from adult human fibroblasts by defined factors. Cell 2007; 131 : 861-872.

31. Wernig M, Meissner A, Foreman R, Brambrink T, Ku M, Hochedlinger $\mathrm{K}$, et al. In vitro reprogramming of fibroblasts into a pluripotent ES-cell-like state. Nature 2007; 448: 318324.

32. Nemeth K, Leelahavanichkul A, Yuen PS, Mayer B, Parmelee A, Doi K, et al. Bone marrow stromal cells attenuate sepsis via prostaglandin $\mathrm{E}(2)$-dependent reprogramming of host macrophages to increase their interleukin-10 production. Nat Med 2009; 15: 42-49.

33. Freudenberg MA, Galanos C. Induction of tolerance to lipopolysaccharide (LPS)-D-galactosamine lethality by pretreatment with LPS is mediated by macrophages. Infect Immun 1988; 56: 1352-1357.

34. DeBoer MD, Scarlett JM, Levasseur PR, Grant WF, Marks DL. Administration of IL-1 beta to the 4th ventricle causes anorexia that is blocked by agouti-related peptide and that coincides with activation of tyrosine-hydroxylase neurons in the nucleus of the solitary tract. Peptides 2009; 30: 210218. 